\title{
Near-Diffraction-Limited Supercontinuum Generation in a Cladding-pumped Nonlinear Fiber Converter
}

\author{
Junhua Ji, Christophe A. Codemard, Andrew Webb, Jayanta K. Sahu and Johan Nilsson \\ Optoelectronics Research Centre, University of Southampton, Southampton, SO17 1BJ, UK \\ juj@orc.soton.ac.uk
}

\begin{abstract}
Through nonlinear scattering including stimulated Raman scattering and beam cleanup, a pulsed multimode pump beam is converted into a nearly diffraction-limited supercontinuum extending from 1 to $2 \mu \mathrm{m}$ in a passive cladding-pumped nonlinear fiber converter.

(C)2010 Optical Society of America

OCIS codes: (190.4370) Nonlinear optics, fibers; (320.6629) Supercontinuum generation.
\end{abstract}

\section{Introduction}

Supercontinuum (SC) generation, as a result of cascaded nonlinear optical effects, has been widely reported and intensively studied since it was discovered by Alfano and Shapiro [1]. With the development of ultra-short pulse diffraction-limited pump lasers, fibers with novel geometries and new materials, SC sources can now cover wavelength from the mid-IR region, e.g., in soft glasses [2], to the UV region, e.g., in silica by tailoring the dispersion of fibers [3]. Notwithstanding this success, power-scaling of diffraction-limited pulsed pump sources is challenging, and this restricts the SC power. However, nowadays the intensities needed to initiate the nonlinear process can be reached also by continuous-wave pump sources. These are easier to power-scale. Therefore, the record average output power reported from SC sources is $50 \mathrm{~W}$, pumped by a single mode $\mathrm{cw} 400 \mathrm{~W}$ industrial fiber laser [4]. Still, this approach presents many challenges, especially in the launch of the pump into a small-core fiber, and altogether, the power conversion efficiency was only $29 \%$ [4].

Here, we propose a radically different, yet simple and effective, approach to generate SC based on our work on cladding-pumped Raman fiber converters and laser sources [5]-[7]. A pulsed multimode pump source is launched into the pump waveguide of a silica-based "passive" double-clad fiber (DCF). In the first step, stimulated Raman scattering then generates a $1^{\text {st }}$ Stokes wave, primarily in the core, which is seeded. With increased pump power, higher-order Stokes waves are generated through cascaded SRS, until the Stokes light reaches the zero dispersion wavelength of the core. Then, a large SC is built up, increasing in spectral width and power as the pump power grows. The SC beam quality is defined by the fiber core geometrical properties. Furthermore, the core dispersion properties can be tailored independently of the cladding design although for efficient Raman conversion core and cladding area must abide some criteria [8].

In this work, we demonstrate a $19 \mathrm{~W}$ average power SC ranging from $1 \mu \mathrm{m}$ to $1.8 \mu \mathrm{m}$ (limited by our instruments) in a $100 \mathrm{~m}$ long DCF with a $20 \mu \mathrm{m}$ diameter core. Given the many benefits of cladding-pumping, including power-handling and easy pump launch, the high efficiency that can be reached with nonlinear scattering even in multimode fiber structures, and the good performance we achieve, we believe this approach offers a simple and cost-effective solution for high-power SC sources.

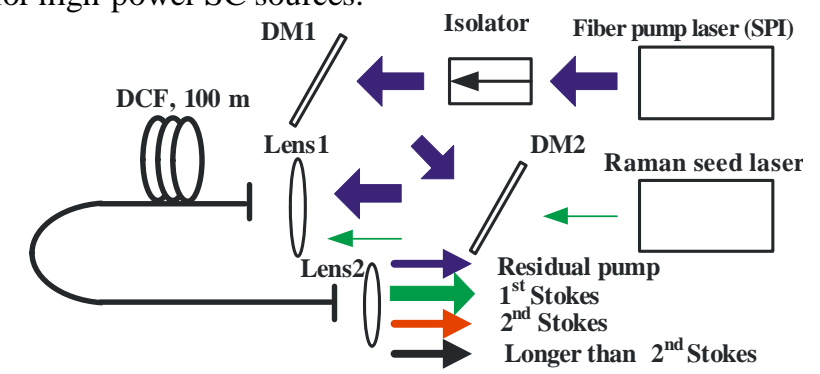

\section{Experimental set-up}

The experimental scheme consists of a low-cost industrial $1064 \mathrm{~nm}$ pulsed fiber laser from SPI laser (SP-30P-0031000) acting as pump source, a $1112 \mathrm{~nm}$ CW seed from a fiber Raman laser, and a $100 \mathrm{~m}$ long piece of passive DCF. See Fig. 1. The DCF has a $20 \mu \mathrm{m}$ diameter, $0.06 \mathrm{NA}$ germanosilicate core, and a $50 \mu \mathrm{m}$ diameter, 0.2 NA silica inner cladding. Thus, the core is multimode for wavelengths below $1.567 \mu \mathrm{m}$. The outer cladding is made of fluorine-doped silica glass and its outer diameter is $160 \mu \mathrm{m}$. The total background loss is $0.24 \mathrm{~dB}$ in this piece of fiber both at the pump and the $1^{\text {st }}$ Stokes wavelength. The pump produces $30 \mathrm{~ns}$ pulses at $150 \mathrm{kHz}$ repetition 
frequency and with peak power up to nearly $10 \mathrm{~kW}$ peak power. The pump is multimode with $\mathrm{M}^{2} \sim 3.2$, while the seed is diffraction-limited. The beams are free-space multiplexed by a dichroic mirror (DM) and launched through a single lens into the DCF. Thus they co-propagate. The seed is launched into the core. Through SRS, the multimode pump amplifies and modulates the seed which becomes pulsed. At the output of the DCF, several DMs are used to separate the residual Raman pump, the $1^{\text {st }}$ Stokes, and higher-order Stokes waves which are generated in the DCF. In this experiment, the launch efficiencies for the pump and seed are respectively $89 \%$ and $92.5 \%$ including seed power launched into the inner cladding. The launched seed power is $88 \mathrm{~mW}$ while the pump power is varied.

\section{Results and discussions}

Figure 2 shows the average output power of the SC source including and excluding the residual pump power against the launched average pump power. The power conversion efficiency is over $70 \%$ with respect to the launched pump power. We measured the output beam quality at different wavelengths using a narrow-band filter. The $\mathrm{M}^{2}$ are 1.69 at $1112 \mathrm{~nm}, 1.55$ at $1178 \mathrm{~nm}$, and 1.58 at $1539 \mathrm{~nm}$. The inset of Fig. 2 depicts the pulse shape at $1539 \mathrm{~nm}$ at $25.4 \mathrm{~W}$ of launched average pump power, showing a pulse durations of $\sim 13 \mathrm{~ns}$.

The output spectrum is shown in Fig. 3 for different pump powers, for this $100 \mathrm{~m}$ long piece and also for a $700 \mathrm{~m}$ long piece. With increased pump power, several discrete order Raman Stokes are generated to about $1233 \mathrm{~nm}$, then the spectra broadens. Calculations with OptiFibre ${ }^{\mathrm{TM}}$ (Optiwave) give a zero dispersion wavelength of around $1278 \mathrm{~nm}$ for the fundamental mode in the core. At the maximum pump power $(8.05 \mathrm{~kW}$ launched peak power), the power density is over $40 \mathrm{~mW} / \mathrm{nm}$ around $1400 \mathrm{~nm}$. This result is close to the record power density of $50 \mathrm{~mW} / \mathrm{nm}$ reported in [4]. In a $700 \mathrm{~m}$ long piece, we extended the SC even further albeit with lower efficiency because of higher losses. We were not able to measure accurately the extension of the SC passed $1750 \mathrm{~nm}$. However, using an un-calibrated Agilent 86140B OSA we were able to verify that the SC extended up to $2 \mu \mathrm{m}$ as shown in the inset of Fig. 3.

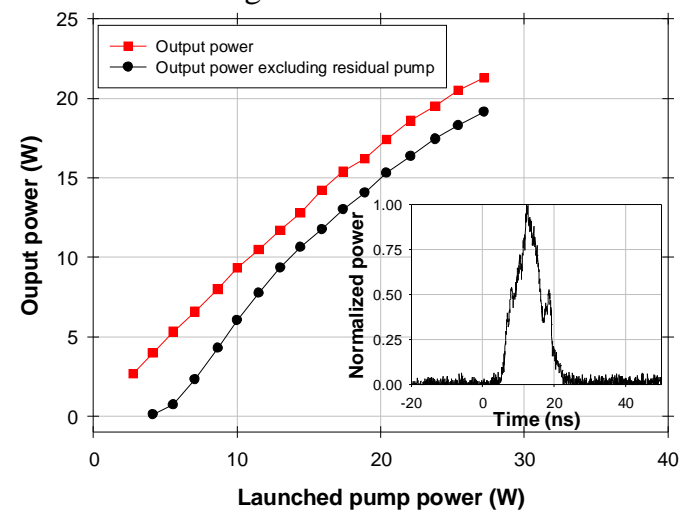

Fig. 2. Average output power vs. average launched pump power. Inset: pulse shape at $1539 \mathrm{~nm}$ at the $25.4 \mathrm{~W}$ launched pump average power.

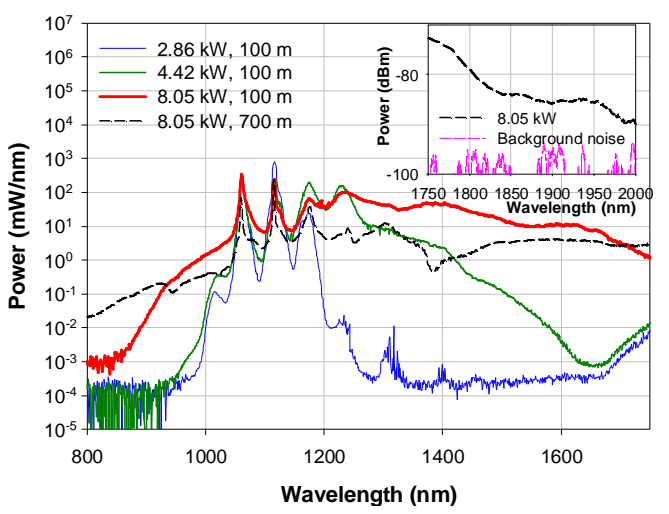

Fig. 3. Output spectra for different launched pump powers. Inset: Spectrum measured by uncalibrated OSA for $700 \mathrm{~m}$ long fiber.

\section{Conclusion}

We have cladding-pumped a $100 \mathrm{~m}$ long nonlinear silica fiber converter with a multimode pump source. This generated a $19 \mathrm{~W}$ average power, pulsed, nearly diffraction-limited supercontinuum with over $70 \%$ conversion efficiency with respect to launch pump power in a large core fiber. We believe this simple approach opens up for substantial further power-scaling of SC sources and that the same method can be readily applied to other type of material such as soft glass fibers which exhibit large nonlinear coefficients and wide transmission window.

Acknowledgments: This work was funded by EPSRC, grant no. EP/E027318/1.

\section{References}

[1] R. R. Alfano and S. L. Shapiro, “Observation of self-phase modulation and small-scale filaments in crystal and glasses,” Phys. Rev. Lett. 24, 592-294 (1970).

[2] J. H. V. Price, et al., "Mid-IP supercontinuum generation from non-silica microstructured fibers," IEEE J. Sel. Top. Quantum Electron. 13, 738-749 (2007).

[3] J. H. V. Price, et al., "UV generation in a pure-silica holey fiber," App. Phys. B 77, 291-298 (2003).

[4] J. C. Travers, et al., "Visible supercontinuum generation in photonic crystal fibers with a $400 \mathrm{~W}$ continuous wave fiber laser," Opt. Express 16, 14435-14447 (2008).

[5] J. Nilsson, et al., "Cladding-pumped Raman fiber amplifier," in Proc. Topical Meeting on Optical Amplifiers and Their Applications, postdeadline paper PDP2, Vancouver, Canada, July 14 - 17 (2002).

[6] C. A. Codemard, et al., "High-power continuous-wave cladding-pumped Raman fiber laser," Opt. Lett., 31, 2290-2292 (2006).

[7] J. Ji, et al., "Beam quality and spectral evolution in large-core cladding-pumped cascaded-Raman fiber converter," in Advanced Solid-State Photonics, Optical Society of America, San Diego, USA 2010 (accepted).

[8] J. Ji, et al. "Analysis of the conversion to the first Stokes in cladding-pumped fiber Raman amplifiers," IEEE J. Sel. Top. Quant. Electron. 15, 129-139 (2009). 